\title{
Liquid phase epitaxial growth of pure and doped GaSb layers: morphological evolution and native defects ${ }^{\dagger}$
}

\author{
P S DUTTA, H L BHAT and VIKRAM KUMAR* \\ Department of Physics, Indian Institute of Science, Bangalore 560012, India \\ * Solid State Physics Laboratory, Lucknow Road, Delhi 110054, India
}

\begin{abstract}
Undoped and Te-doped gallium antimonide (GaSb) layers have been grown on $\mathrm{GaSb}$ bulk substrates by the liquid phase epitaxial technique from $\mathrm{Ga}$-rich and $\mathrm{Sb}$-rich melts. The nucleation morphology of the grown layers has been studied as a function of growth temperature and substrate orientation. MOS structures have been fabricated on the epilayers to evaluate the native defect content in the grown layers from the $C-V$ characteristics. Layers grown from antimony rich melts always exhibit $p$-type conductivity. In contrast, a type conversion from $p$ - to $n$-was observed in layers grown from gallium rich melts below $400 \mathrm{C}$. The electron mobility of undoped $n$-type layers grown from $\mathrm{Ga}$-rich melts and tellurium doped layers grown from $\mathrm{Sb}$ - and $\mathrm{Ga}$-rich solutions has been evaluated.
\end{abstract}

Keywords. Liquid phase epitaxy; growth: GaSb.

\section{Introduction}

Liquid phase epitaxy (LPE) has proved to be a versatile technique to grow semiconductor layers for material investigations and device applications in the past (Kuphal 1991). Today, inspite of availability of highly sophisticated epitaxial technologies like MBE and MOCVD, the exploration and development of many devices are still carried out using LPE. This is essentially due to simplicity of the technique and high crystalline quality of grown films. Until now, research in III-V compounds for optoelectronic devices has mainly been focussed on GaAs and InP (Kuphal 1991). However, recent developments in non-silica fibre technology have urged the need for newer materials operating in the $2.5 \mu \mathrm{m}$ regime (Tran et al 1984; France et al 1986). The ternary and quaternary alloys lattice matched to GaSb suit the specifications of sources and detectors for this region (Milnes and Polyakov 1993). At present, GaSb technology is in its infancy and even the growth of reliable and reproducible GaSb epitaxial films on bulk substrates has not been optimized. In a recent investigation (Dutta et al 1995), we have addressed several growth related problems during liquid phase epitaxy of $\mathrm{GaSb}$ and its effect on the optical and $p-n$ junction properties of layers. A further extension of this study to understand the nucleation morphologies of layers as well as the investigation relating to native defects are presented here. In general, systematic electrical characterization of $\mathrm{GaSb}$ epilayers by Hall measurements has been previously impeded by the lack of GaSb semi-insulating substrate. Also, low Schottky barrier height on $p$-GaSb has greatly obstructed the evaluation of the electrically active defects in as-grown undoped $p$-type layers by the $C-V$ technique. During the course of this work, we have successfully overcome these problems by fabricating MOS structures on the epilayers. The net impurity concentration in the grown layers has been evaluated from the $C-V$ characteristics of the MOS diodes. This technique has not been

\footnotetext{
${ }^{+}$Paper presented at the poster session of MRSI AGM VI. Kharagpur. 1995
} 
employed previously for characterization of $\mathrm{GaSb}$ and will be discussed in detail in this paper.

\section{Experimental}

The undoped and Te-doped LPE layers used for our studies were grown by the sliding boat technique. The bulk substrates used were undoped $p$-GaSb grown in our laboratory by the vertical Bridgman technique (Dutta et al 1994). The room temperature hole concentration in the bulk samples was $\sim 10^{17} \mathrm{~cm}^{-3}$. Growth from Ga-rich as well as $\mathrm{Sb}$-rich melts were carried out in the temperature range of $300-550^{\circ} \mathrm{C}$ and $630-680^{\circ} \mathrm{C}$ respectively. The details of the growth experiments have been published recently (Dutta et al 1995). The surface morphology of the layers were examined using the LeitzOrthoplan microscope in reflection mode essentially to study the mechanism of growth from Ga-rich and Sb-rich solutions. The conductivity type of the grown layers were evaluated by the hot-point probe technique. Wet oxygen annealing was carried out to obtain oxide layers on the $\mathrm{GaSb}$ epilayers. Oxidation at $400^{\circ} \mathrm{C}$ for $200 \mathrm{~min}$ resulted in highly uniform oxide layers of $\sim 1000 \AA$ thickness. Prior to oxidation, the back ohmic contacts to the GaSb substrates were provided by the Au:Zn (98:2) eutectic mixture, thermally deposited and annealed at $300^{\circ} \mathrm{C}$ for $2 \mathrm{~min}$. The metal contact on the oxide layer was provided by thermal evaporation of aluminium at $10^{-6}$ torr. For the Hall measurements on the $n$-type layers, the ohmic contacts were prepared by indium.

\section{Results and discussion}

\subsection{Nucleation morphology}

The surface morphology of LPE grown layers depends on several factors like substrate preparation and orientation, extent of thermal decomposition of the substrate prior to growth, inadequate wetting and non-uniform meltback, phase changes due to high impurity levels, degree of melt supersaturation, constitutional supercooling, etc (Mattes and Route 1974; Mottram and Peaker 1974). However, in any particular growth system, simple working tolerances can be established to ensure reproducible growth of high quality layers. Our previous studies (Dutta et al 1995) of growth by equilibrium cooling technique on $7^{\circ}$ off (111) oriented substrates from Ga-rich solutions at temperatures below $400^{\circ} \mathrm{C}$ gave layers with large number of islands, fairly deep undulations and gallium inclusions across the surface (refer figure 1a in Dutta et al 1995). The poor morphology on highly misoriented substrate is typical for all LPE grown layers (Saul and Roccasecca 1973). In our case, the problem was probably further enhanced by inadequate wetting and presence of thin oxide film on the substrate due to inefficient reduction of oxides by hydrogen at low temperatures. However, above $\sim 500^{\circ} \mathrm{C}$, planar uniform layers could be grown. An indepth investigation of the morphological evolution from the poor patchy surfaces obtained at low growth temperatures to smooth planar ones at higher temperatures would help in understanding the basic crystal growth phenomenon. The morphological aspects of layers grown at intermediate temperatures are discussed below.

Figure la shows the morphology of layer grown at $450^{\circ} \mathrm{C}$. The layer exhibits ripples or usual tide-like pattern, known as terraces. With increase in growth temperature, the 

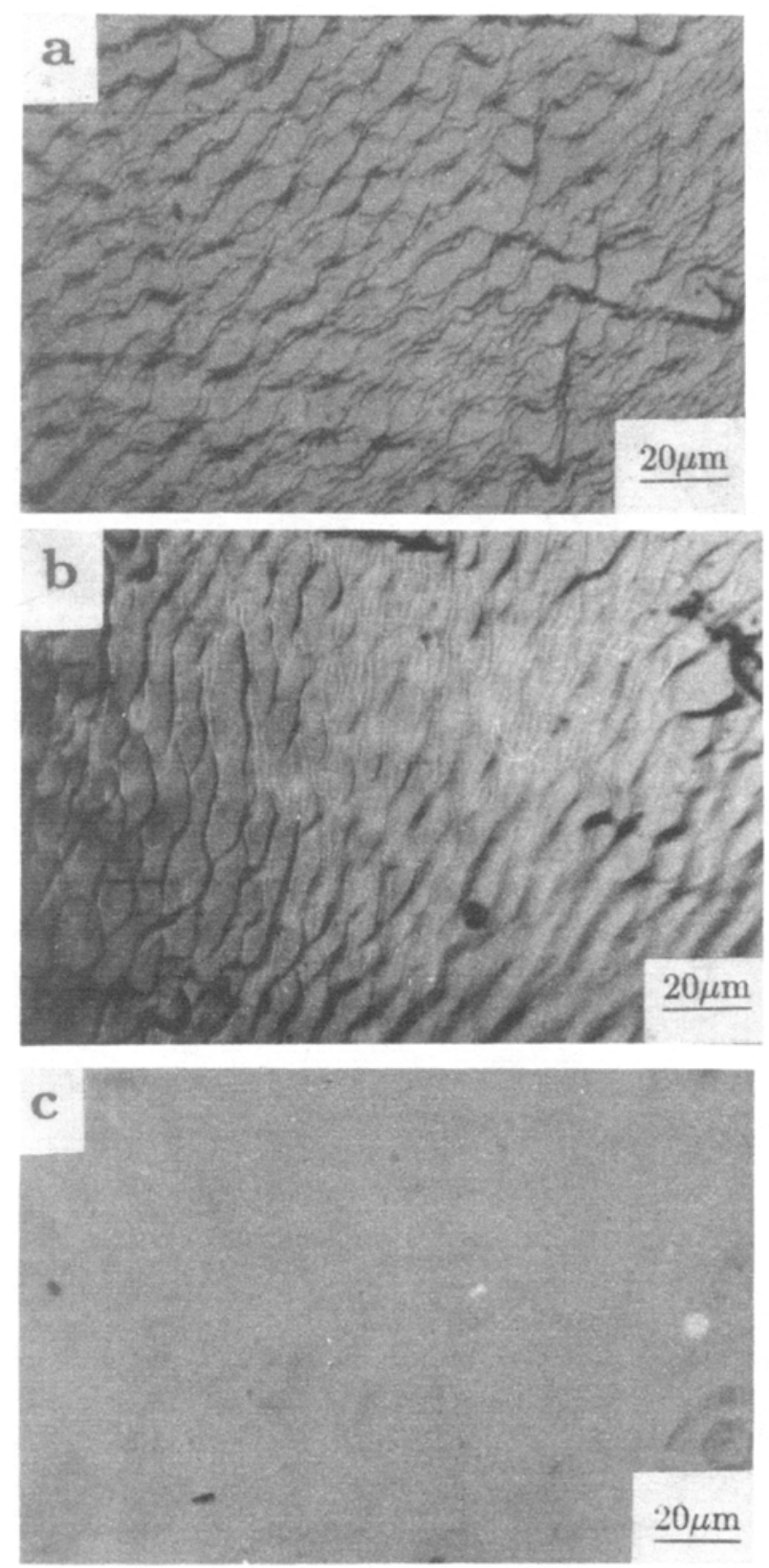

Figure 1. Surface morphologies of $\mathrm{GaSb}$ epilayers grown from $\mathrm{Ga}$-rich melts. The layer thickness is given in square brackets. (a) on $7^{\circ}$ off(111) substrates at $450^{\circ} \mathrm{C}[14 \mu \mathrm{m}]$, (b) on $7^{\circ}$ off (111) substrates at $500^{\circ} \mathrm{C}[16 \mu \mathrm{m}]$ and (c) on $(110)$ substrates at $550^{\circ} \mathrm{C}[12 \mu \mathrm{m}]$.

width of the terrace decreases (see figure 1b). Ultimately, at temperatures around $500^{\circ} \mathrm{C}$, the layers revealed flat surfaces. The uniformity of the surface was even better if a (110) or (100) substrate was used instead of a misoriented (111). Figure 1c shows the smooth mirror-like morphology of a layer grown at $550^{\circ} \mathrm{C}$ on $(110)$ oriented substrate. 

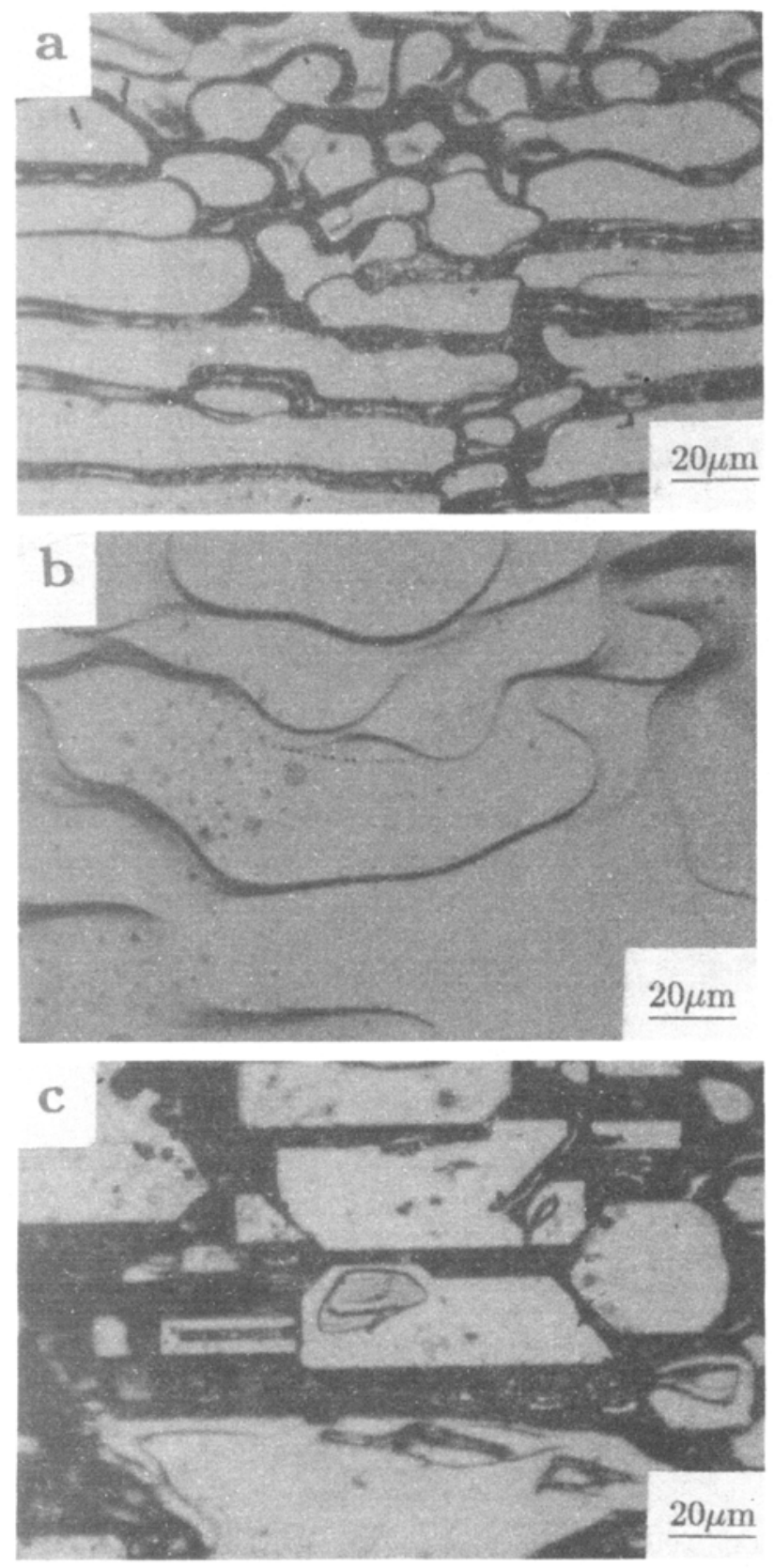

Figure 2. Surface morphologies of GaSb epilayers on $7^{\circ}$ off (111) substrates grown from $\mathrm{Sb}$-rich melts at various temperatures. The layer thickness is given in square brackets. (a) $640^{\circ} \mathrm{C}[10 \mu \mathrm{m}]$, (b) $660^{\circ} \mathrm{C}[9 \mu \mathrm{m}]$ and (c) $680^{\circ} \mathrm{C}[12 \mu \mathrm{m}]$.

The presence of gallium inclusions in the layers grown at low temperatures can be due to constitutional supercooling or cellular convection in the growth solution (Small and Crossley 1974). The disappearance of irregularities at high growth temperatures is 
attributed to the increase in thermal gradient across the melt-substrate interface. This reduces the terrace width by reducing the diffuse interface region, and thereby increasing the nucleation density (Mattes and Route 1974). At the same time, we do not rule out the possibility of terrace formation due to submicroscopic terrace structure left after the usual polishing and etching at the surface of a misoriented substrate as suggested by Bauser et al (1974). From technological point of view, perturbations like terraces and layer discontinuity can deleteriously affect the device characteristics by causing optical scattering, increasing threshold and dark current levels, degrading signal to noise ratios etc. Hence, these morphologies are undesirable. By imposing sufficient initial supercooling in the melt prior to contact with the substrate and during growth thereby avoiding constitutional supercooling the terrace formation can be suppressed. This has been illustrated by us in the case of growth by step-cooling, super-cooling and two-phase solution growth techniques earlier (Dutta et al 1995).

Growth from Sb-rich melts exhibited interesting nucleation and morphological aspects. Our initial experiments revealed that growth from Sb-rich melts always resulted in inferior surface morphology to those grown by Ga-rich melts at all growth temperatures and irrespective of the substrate orientation. Systematic studies were carried out to understand the morphological aspects of layers grown at different temperatures and are discussed below. Figure 2a shows the layer grown on $7^{\circ}$ off oriented (111) substrate at $630^{\circ} \mathrm{C}$ by the equilibrium cooling technique. Large number of ridges and islands can be seen. The poor quality of the layer is due to high precipitate formation and the sticking problem after growth. On the other hand, at high temperatures $\sim 670^{\circ} \mathrm{C}$, there is volatilization of antimony from the melt as well as from the substrate (prior to growth) which result in layers with unevenly distributed flat plateaux separated by inclusion valleys as shown in figure $2 \mathrm{c}$. Growth beyond $680^{\circ} \mathrm{C}$ is not possible due to the softening of the substrate. Woelk and Benz (1974) explained the high precipitate formation during growth from Sb-rich melts in terms of the slope of liquidus curve of $\mathrm{Ga}-\mathrm{Sb}$ phase diagram on the $\mathrm{Sb}$-rich side. High growth rate is expected as a result of this and hence unconttolled growth occurs. The morphology of continuous layer as shown in figure $2 b$ for an intermediate growth temperature sheds light on the nucleation properties of the layer grown from Sb-rich melts. It resembles that of a nucleation on a terraced surface with faceted upper surface as has been observed previously by Small et al (1977) in the case of AlGaAs layers on GaAs substrates. This indicates a non-zero contact angle between the epilayer and substrate under the conditions of growth on misoriented substrates. It might be argued that the non-zero contact angle in our case could be due to thin oxide coating on the melt which is more probable in Sb-rich solutions. In figure $2 b$, a sharp distinction between the upper and lower regions can be seen which are terraced and smooth respectively. The appearance of these regions may be due to the following. It is seen that the top of each segment is faceted and when two segments coalesce due to one covering the width of the terrace, then the growth step changes its slope and advances rapidly over the surface leading to a smooth region. The absence of such facets in layers grown from Ga-rich melts is attributed to contact angle being less than the misorientation angle. Thus, the difference in the nucleation properties of layers grown from Ga-rich and Sb-rich melts arises from the contact angle at the epilayer-substrate junction. This fundamental difference is highly detrimental for device applications. Even though terraces are present in layers grown from both Ga-rich and Sb-rich melts, the epilayers grown from Sb-rich melts additionally exhibit facets. Since facets act as regions of high impurity 


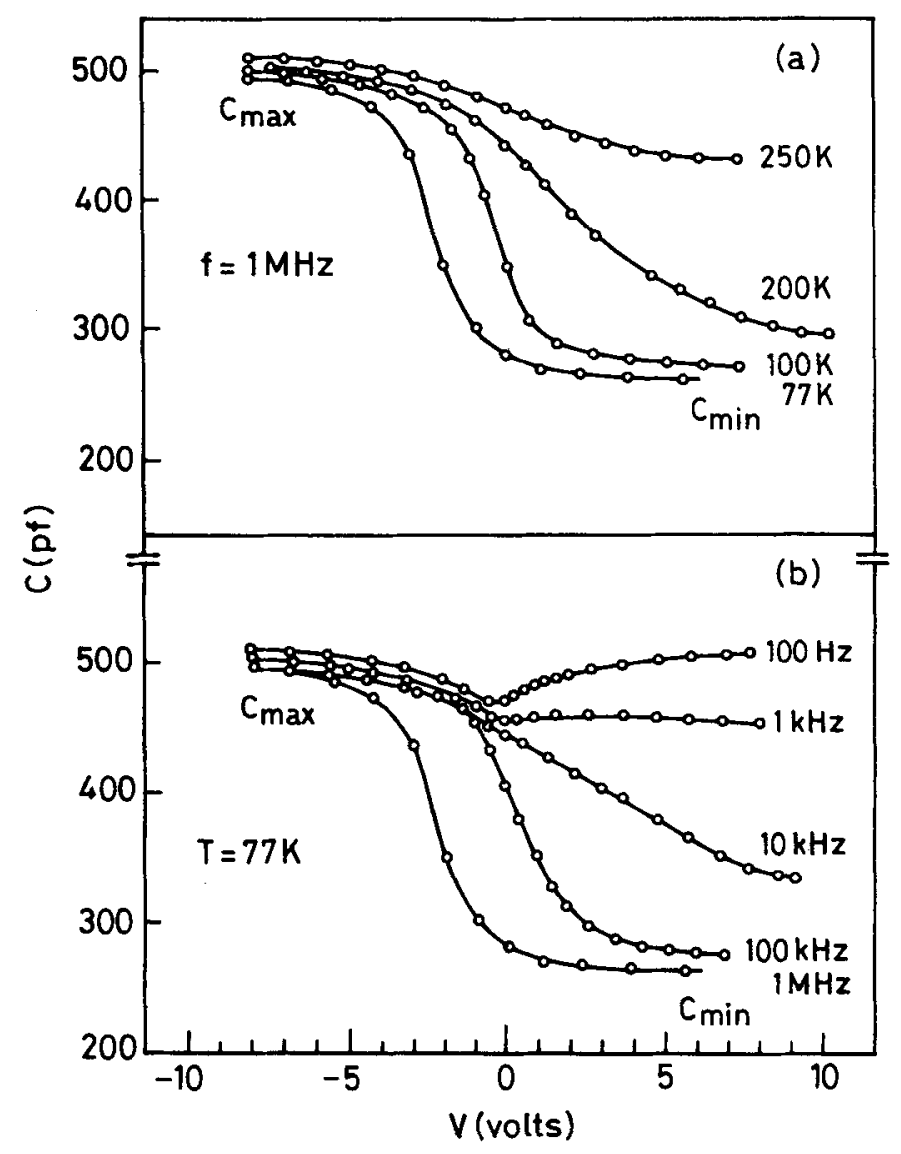

Figure 3. (a) Temperature dependence of high frequency $C \sim V$ characteristics and (b) frequency dependence of the $C-V$ characteristics of the MOS diodes.

concentration, it leads to inhomogeneous impurity distribution in the grown layers thus hindering device performance.

\subsection{Native defect and mobility analyses}

The evaluation of net defect concentration from the $C-V$ characteristics of the MOS structure is accurate, provided the influence of the interface states during the measurements is eliminated (Sze 1981). Hence, initially the steady-state $C-V$ characteristics were measured at various temperatures and signal frequencies to find the optimum conditions under which the influence of the interface states are negligible. The results are shown in figures $3 a$ and $b$. At room temperature, the $C-V$ curve is found to be too flat to reflect the impurity concentration in the bulk probably due to the high density of interface states (see figure 3a). However, with decrease in temperature, the contribution from the interface states reduces continuously and below $100 \mathrm{~K}$, the capacitance saturates properly. At $77 \mathrm{~K}$, the interface states are mostly frozen and an expected high-frequency $C-V$ characteristic is observed. At these low temperatures, it took several minutes to obtain the steady-state capacitance. Figure 3 b shows the test-signal 
frequency dependence of the $C-V$ characteristics at $77 \mathrm{~K}$. As is clear from the figure, that at high frequency $\sim 1 \mathrm{MHz}$, the minimum capacitance saturates well. Thus it can be concluded from the above measurements that the steady-state $C-V$ characteristics at $1 \mathrm{MHz}$ and at $77 \mathrm{~K}$ is suitable to obtain the maximum and the minimum capacitance with negligible contribution from the interface states.

The $C-V$ measurements were then performed on all the grown epilayers with the above optimized conditions. From the maximum $\left(C_{\max }\right)$ and minimum $\left(C_{\min }\right)$ capacitances of the steady-state high-frequency $C$ - $V$ curve, the net impurity concentration can be calculated using the following equation (Sze 1981):

where

$$
\frac{C_{\min }}{C_{\max }}=\frac{1}{1+\left(C_{\max } W_{\mathrm{m}}\right) /\left(\varepsilon_{0} \varepsilon_{\mathrm{s}} S\right)},
$$

$$
W_{\mathrm{m}}=\left\{4 \varepsilon_{0} \varepsilon_{\mathrm{s}} k_{\mathrm{B}} T \ln \left[\left(N_{\mathrm{A}}-N_{\mathrm{D}}\right) / n_{\mathrm{i}}\right] / q^{2}\left(N_{\mathrm{A}}-N_{\mathrm{D}}\right)\right\}^{1 / 2},
$$

where $\varepsilon_{0}$ is the dielectric constant of free space, $\varepsilon_{\mathrm{s}}$ the static dielectric constant of the semiconductor, $S$ the area of the metal contact, $k_{\mathrm{B}}$ Boltzmann's constant, $T$ the absolute temperature of the sample in degrees Kelvin, $N_{\mathrm{A}}$ and $N_{\mathrm{D}}$ are the acceptor and donor concentration, respectively and $n_{\mathrm{i}}$ is the intrinsic carrier concentration.

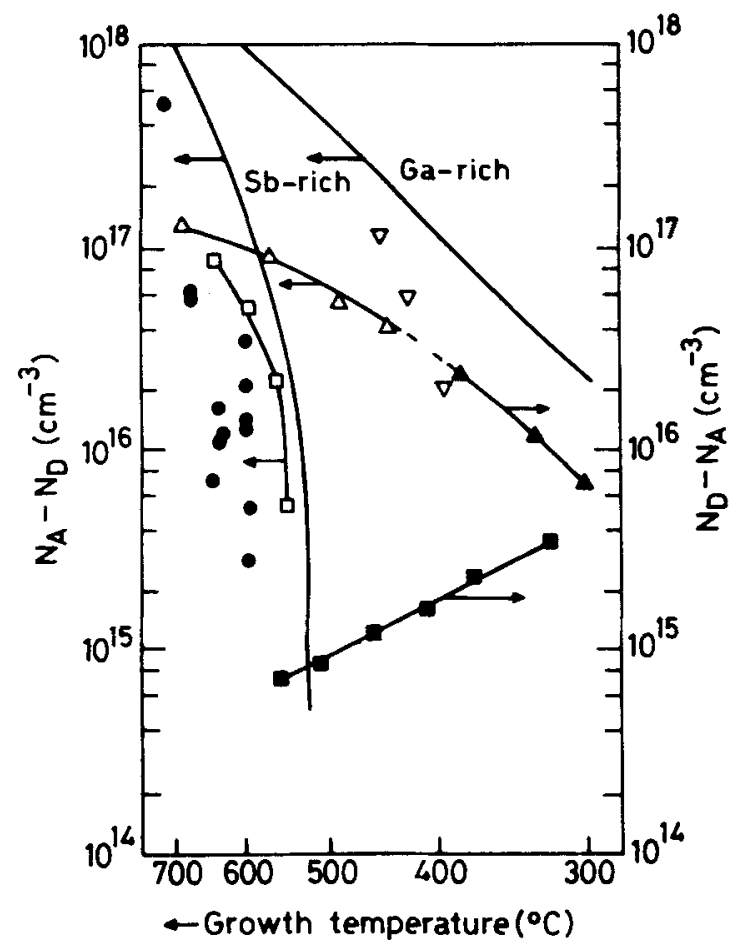

Figure 4. Theoretically calculated and experimentally measured net acceptor and donor concentrations for GaSb epilayers grown at various temperatures from $\mathrm{Ga}$ - and $\mathrm{Sb}$-rich melts. Theoretically calculated values (- - - ) ; Experimental data for p-GaSb grown from Ga-rich $(\nabla)$ and Sb-rich (O) melts by Anayama $e t$ al (1990). Experimental data (this work) for undoped $p-(\Delta)$ and $n$-( $\Delta)$ type layers grown from Ga-rich melts, undoped $p$-type layers grown from Sb-rich melts $(\square)$ and Te-doped layers grown from Ga-rich melts ( $\square$ ). 
The net impurity concentration evaluated from the $C-V$ characteristics in the grown layers at various temperatures are shown in figure 4 . The experimental data of net acceptor concentration in layers grown from Ga-rich and Sb-rich melts by Anayama et al (1990) and the theoretical results of Ichimura et al (1990) are also included in the figure for comparison. The theoretical lines of the net defect concentration shown here have been evaluated from the individual native defect concentrations (vacancies and antisites) found by Ichimura et al (1990). As can be seen from the figure, the theoretical calculation predicts that layers grown from both Ga-rich and $\mathrm{Sb}$-rich melts always exhibit $p$-type conduction irrespective of the growth temperature with the net acceptor concentration decreasing with decrease in growth temperatures. Nevertheless for the Sb-rich melts, the decrease in net acceptor concentration is much faster than that for the Ga-rich case. It is worth pointing out that the experimental results follow the trend predicted by the theory but differ in numerical values. This difference can be attributed to the formation of complex defects like $\mathrm{V}_{\mathrm{Ga}} \mathrm{Ga}_{\mathrm{sb}}$ (van Maaren 1966) which has not been taken into account in the theoretical model. Moreover, the defect concentration has been calculated at the growth temperature. But, some defects may get annealed out during the post growth cooling cycle. Referring to the figure, our studies also show that it is possible to obtain undoped $n$-type layers at low temperature from $\mathrm{Ga}$-rich melts (shown by filled triangles). From the figure, it can be inferred that the type conversion takes place around $400^{\circ} \mathrm{C}$. Previously, Miki et al (1974) also obtained undoped $n$-type layers from Ga-rich melts at $400^{\circ} \mathrm{C}$. The $p$ to $n$-conversion is attributed to the presence of extrinsic donors in the layers whose effect can be seen only at low growth temperatures when the compensating native acceptor concentration is low. Such type conversion is not predicted from the theoretical calculations as the theory has not taken the extrinsic impurities into account. It is also worth mentioning that the variation of net donor concentration with growth temperature is different for the undoped and Te-doped layers. In case of undoped layers, the net donor concentration decreases with decreasing growth temperature, whereas, the opposite trend is observed in the Te-doped layers. This difference is attributed to the change in distribution coefficient of the extrinsic impurities with temperature and the resulting degree of compensation. Silicon and oxygen are two unintentional impurities usually present in the LPE layers. While Si gives $p$-type layers with heavy compensation due to its amphoteric nature, oxygen is a donor. Previously, Takeda et al (1985) found that the distribution coefficient of Si increases with decrease in growth temperature. Thus the incorporation of $\mathrm{Si}$ increases and the net donor concentration decreases with decrease in growth temperature. The monotonic increase in the net donor concentration in Te-doped layers with decrease in growth temperature for the same mole fraction of $\mathrm{Te}$ in the melt may arise from the difference in distribution coefficients of the donors and acceptors with temperature below $400^{\circ} \mathrm{C}$ and due to decrease in native acceptor concentration above $400^{\circ} \mathrm{C}$. Thus it may be concluded that the increase in distribution coefficient of Te with decreasing temperature below $400^{\circ} \mathrm{C}$ is more than that of the compensating acceptors.

Figure 5 shows the electron mobility at $77 \mathrm{~K}$ as a function of growth temperature for Te-doped $n$-type layers grown from $\mathrm{Ga}$-rich and $\mathrm{Sb}$-rich melts as well as undoped $n$-type layers grown from Ga-rich melts. It should be noted that both the net donor concentration (see figure 4) as well as the mobility increase with decrease in growth temperature. This peculiar electrical characteristics of $n$-GaSb has also been observed previously (Baxter et al 1967). This occurs at low doping levels and is due to the effect of 


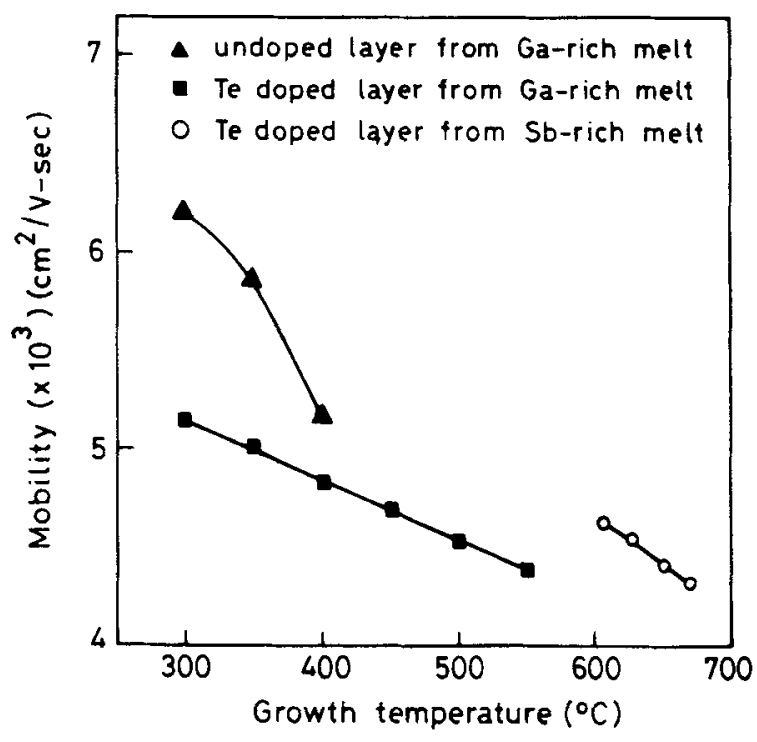

Figure 5. Electron mobility of various GaSb layers at $77 \mathrm{~K}$.

heavy compensation on Coulomb or impurity scattering. This do not happen in case of layers with higher doping levels as in the case of undoped $n$-type layers. As expected, the mobility increases with decreasing growth temperature which is due to decrease in degree of compensation by the native acceptors. Also. for the same values of mobilities, a lower growth temperature is required for the Ga-rich case than the $\mathbf{S b}$-rich melts. This clearly indicates that the defect formation in $\mathrm{GaSb}$ is monitored by the vacant antimony site (Anayama et al 1990). Lastly, it should be pointed out that the undoped $n$-type layers (grown from Ga-rich melts) possess higher mobility than those doped with tellurium at the same growth temperature which is understandable.

\section{Conclusions}

In conclusion, the nucleation morphologies of $\mathrm{GaSb}$ layers grown from $\mathrm{Ga}$-rich and Sb-rich melts have been investigated. The fundamental difference in the two cases is that, while from Sb-rich melts faceted growth on highly terraced surface occurs. in Ga-rich melts the growth occurs in fine terraces without facets. This is attributed to the difference in the contact angle between the epilayer and substrate in the two cases. Further, we have been able to systematically measure the native defect concentration in the epilayers grown from $\mathrm{Ga}$ - and $\mathrm{Sb}$-rich melts. A comparison of the morphological and electrical properties of the grown layers shows that even though from native defect point of view the Sb-rich layers would be desirable. the main drawbacks of these layers are the poor surface morphology and the presence of facets which will lead to inhomogeneous impurity distribution. Further work in improving the quality of surface morphology such as by rare-earth doping which has been previously used for GaAs and InP (Bantein et al 1987) is worth pursuing. 


\section{Acknowledgements}

One of the authors (PSD) would like to thank CSIR, New Delhi for the award of a senior research fellowship. We also wish to acknowledge the financial support from the Indian Telephone Industries Limited, Bangalore.

\section{References}

Anayama C, Tanahashi T, Kuwatsuka H, Nishiyama S, Isozumi S and Nakajima K 1990 Appl. Phys. Letts. 56239

Bantein F, Bauser E and Weber J 1987 J. Appl. Phys. 612803

Bauser E, Frick M, Loechner K S, Schmidt L and Ulrich R 1974 J. Crystal Growth 27148

Baxter R D, Reid F J and Beer A C 1967 Phys. Rev. 162718

Dutta P S, Sangunni K S, Bhat H L and Kumar V 1994 J. Crystal Growth 14144

Dutta P S, Koteswara Rao K S R, Bhat H L, Gopalakrishna Naik K and Kumar V 1995 J. Crystal Growth 14214

France P W, Carter S F, Moore M W and Williams J R 1986 SPIE 61851

Ichimura M, Higuchi K, Hattori Y, Wada T and Kitamura N 1990 J. Appl. Phys. 686153

Kuphal E 1991 Appl. Phys. A52 380

van Maaren M H 1966 J. Phys. Chem. Solids 27472

Mattes B L and Route R K 1974 J. Crystal Growth 27133

Miki H, Segawa K and Fujibayashi K 1974 Jpn J. Appl. Phys. 13203

Milnes A G and Polyakov A Y 1993 Solid State Electronics 36803

Mottram A and Peaker A R 1974 J. Crystal Growth 27193

Saul R H and Roccasecca D D 1973 J. Appl. Phys. 441983

Small M B and Crossley I 1974 J. Crystal Growth 2735

Small M B, Blum J M and Potemski R M 1977 Inst. Phys. Conf. Ser, 33a 9

Sze S M 1981 Physics of semiconductor devices (New York: Wiley) Ch. 7

Takeda Y, Noda S and Sasaki A 1985 J. Appl. Phys. 571261

Tran D C, Siegel G H Jr. and Bendow B 1984 Lightwave Technol. LT-2 536

Woelk C and Benz K W 1974 J. Crystal Growth 27177 